\title{
Análisis de Modelos Rivales Unidimensionales y Bidimensionales de la Escala Breve de Autocontrol en Estudiantes Universitarios Argentinos
}

\author{
Sebastian Jesús Garrido ${ }^{12}$, Valeria Morán², Ana Estefanía Azpilicueta ${ }^{2}$, Franco \\ Cortez $^{1}$, Karin Arbach ${ }^{12}$ y Marcos Cupani ${ }^{12}$
}

\begin{abstract}
RESUMEN
La Escala Breve de Autocontrol (BSCS, por sus siglas en inglés) es un instrumento de autoinforme ampliamente utilizado para evaluar el autocontrol. Sin embargo, existen desacuerdos respecto a su estructura interna. En este trabajo se realizaron una serie de análisis factoriales confirmatorios (AFC) con el fin de comparar estructuras uni y bidimensionales en una muestra de 848 estudiantes universitarios argentinos (663 mujeres y 185 varones), con un rango etario comprendido entre 18 y 30 años. De manera complementaria, se evaluó la consistencia interna, el ajuste de los modelos teóricos propuestos sobre la estructura interna de la escala, y se realizó un estudio de evidencia de validez de grupos contrastados. A partir de los resultados, se concluyó que el modelo post hoc unidimensional propuesto por Tangney, Baumeister y Boone (2004) es la opción que mejores propiedades psicométricas presentó para ser utilizado con fines de investigación en muestras de estudiantes universitarios argentinos.

Palabras clave: autocontrol, Escala Breve de Autocontrol (BSCS), modelos rivales, análisis factorial confirmatorio, consumo de sustancias.
\end{abstract}

\section{Analysis of Unidimensionality and Bidimensionality Rivals Models of Brief Self-Control Scale in Argentinean University Students}

\begin{abstract}
The Brief Self-Control Scale (BSCS) is a broad used measure to assess self-control. However, there is a lack of agreement about its internal structure. Therefore, in this study we carried several confirmatory factorial analysis (CFA) to compare unidimensionality and bidimensionality structures, on a sample of 848 Argentinian college students (663 female and 185 male), aged between 18 And 30 years old. Additionally, we made internal consistency analysis, and we obtained the contrast group validity. Based on the results obtained, we concluded that the unidimensional post-hoc model designed by Tangney, Baumeister and Boone (2004) possess the best psychometric properties, therefore is can be considered a valid and reliable instrument to use in academic research.
\end{abstract}

Keywords: self-control, Brief Self Control Scale (BSCS), rivals models, confirmatory factorial analysis, substance consumption.

El concepto de autocontrol ha sido ampliamente estudiado en el campo de la psicología, psiquiatría y sociología (Duckworth \& Kern, 2011). Se destaca particularmente la influencia, tanto positiva como negativa, que este

${ }^{1}$ Universidad Nacional de Córdoba, Facultad de Psicología, Argentina; sebajgarrido@gmail.com

${ }_{2}$ Instituto de Investigaciones Psicologicas (IIPSI)-CONICET, Argentina. 
constructo ha demostrado tener en la calidad de vida de las personas y de su entorno (De Ridder, Lensvelt-Mulders, Finkenauer, Stok \& Baumeister, 2012). En efecto, se ha observado que un elevado nivel de autocontrol se asocia con un estilo de vida exitoso (Tangney, Baumeister, \& Boone, 2004), un buen rendimiento académico (Job, Friese, \& Bernecker, 2015) y patrones de alimentación saludables (Will Crescioni et al., 2011). Por otro lado, niveles bajos de autocontrol se asocian con alteraciones en la conducta, tales como desórdenes de alimentación (Konttinen, Haukkala, Sarlio-Lähteenkorva, Silventoinen, \& Jousilahti, 2009), consumo excesivo de alcohol (Visser, De Winter, Veenstra, Verhulst, \& Reijneveld, 2013) y comportamiento violento (Turanovic, Reisig, \& Pratt, 2015).

Desde la postura teórica contemporánea del autocontrol este concepto se define como la regulación de los impulsos para el logro de objetivos a largo plazo. El autocontrol se manifiesta en situaciones en las cuales el sujeto debe realizar una elección entre dos opciones asimétricas, es decir, entre una gratificación inmediata y una gratificación a largo plazo, la cual supone consecuencias de mayor importancia para el individuo. En este contexto de decisión, el autocontrol es activado por el propio sujeto con el objetivo de alcanzar esta meta a largo plazo (Duckworth, Gendler, \& Gross, 2016). En esta misma línea, Tangney et al. (2004) se refieren al autocontrol como la habilidad de una persona para invalidar, inhibir y regular impulsos socialmente inaceptables e indeseables, que de ser expresados llevarian a una gratificación inmediata, pero que al ser inhibidos permiten el logro de resultados favorables en el futuro.

En cuanto a la estimación de este constructo, una de las escalas más ampliamente utilizadas es la Escala Abreviada de Autocontrol (BSCS; Tangney et al., 2004), la cual posee 13 items y permite evaluar el autocontrol de forma unidimensional. Cabe mencionar que es la versión breve de la Escala de Autocontrol (SCS, por sus siglas en inglés) desarrollada inicialmente por los mismos autores y que permite medir cinco factores específicos (Autodisciplina, Hábitos Deliberados/ No Impulsivos, Hábitos Saludables, Ética de Trabajo y Confiabilidad). La fiabilidad de ambas versiones es satisfactoria (alfa de Cronbach de .89 para SCS y de .85 para la BSCS) así como también la correlación entre ambas $(r=.93)$. La BSCS ha sido adaptada a diferentes culturas e idiomas y posee amplia recepción en el campo de la investigación psicológica (Ludwig et al., 2013; Malouf, Stuewig, \& Tangney, 2012; Prado, Treeby, \& Crowe, 2015). Sin embargo, los resultados obtenidos en diversos estudios psicométricos presentaron diferencias en relación con su estructura factorial -unidimensional o bidimensional- y los items que conforman cada factor (Lindner, Nagy, \& Retelsdorf, 2015; Maloney, Grawitch, \& Barber, 2012; Morean et al., 2014).

Los autores que avalan la unidimensionalidad de la escala (Tangney et al., 2004) sostienen que la BSCS es una medida global que permite valorar el autocontrol, mientras que los autores que abogan por la multidimensionalidad (e.g., De Ridder, De Boer, Lugtig, Bakker, \& Van Hooft, 2011; Ferrari, Stevens, \& Jason, 2009; Maloney et al., 2012; Morean et al., 2014) proponen que la escala está compuesta por dos factores que explican la totalidad de la varianza. Así, De Ridder et al. (2011) identificaron dos factores, Inhibición e Indicación. El primero se refiere a la capacidad del individuo para inhibir los impulsos inmediatos, mientras que el segundo factor se refiere a la iniciación de un comportamiento dirigido a metas. En esta misma línea, Ferrari et al. 
(2009), a partir del análisis factorial exploratorio (AFE), obtuvieron una solución de dos factores, Autodisciplina General, definido como aquellos patrones de comportamiento general tendientes a resistir tentaciones, y Control de Impulsos, definido como la resistencia a las recompensas a corto plazo con el fin de lograr objetivos a largo plazo. En un estudio más reciente, Maloney et al. (2012) utilizaron AFE y análisis factorial confirmatorio (AFC), con los cuales obtuvieron una solución factorial de dos dimensiones. La primera dimensión, Impulsividad, hace referencia al comportamiento espontáneo que es guiado por la intuición y la heurística. La segunda dimensión, Restricción, hace referencia a la tendencia a manifestar un comportamiento deliberado o planificado. Por su parte, Morean et al. (2014) utilizaron el mismo procedimiento metodológico que Maloney et al. (2012) y reportaron una solución factorial compuesta por el factor Autodisciplina y el factor Control de los Impulsos, cuyas definiciones son similares a las propuestas por Maloney et al. (2012).

Como se expuso hasta aquí, y considerando que no existe consenso entre los modelos teóricos propuestos para determinar la configuración interna de la BSCS, el presente trabajo tiene como objetivo determinar cuál es la estructura factorial más adecuada para ser utilizada en estudiantes universitarios de la Ciudad de Córdoba (Argentina). Para esto, se compararán los modelos propuestos utilizando la estrategia de modelos rivales con datos de una misma muestra de estudiantes. Cuando existen modelos alternativos para representar una misma situación sin conocer cuál proporciona un mejor nivel de ajuste, la estrategia de modelos rivales proporciona las líneas básicas para realizar esta comparación y seleccionar el más idóneo (Cupani, 2012). Por otro lado, teniendo en cuenta que las conductas de consumo de sustancias como el alcohol o las drogas se encuentran relacionadas con el autocontrol que el sujeto posea (Duckworth \& Kern, 2011), se conformaron dos grupos diferenciados entre sí, de acuerdo con la presencia o ausencia de consumo de sustancia, para evaluar si existía diferencia en el autocontrol entre los participantes que consumían y entre los que no.

\section{MÉTODO}

\section{Participantes}

La muestra estuvo compuesta por 848 participantes, de los cuales el $78 \%$ (663) eran mujeres y $22 \%$ (185) hombres, con edades comprendidas entre 18 y 30 años. Cabe mencionar que la proporción de hombres y mujeres que componen la muestra no dista sustancialmente de la observada en la población estudiantil de la universidad, la cual hasta el año 2016 registraba 118.949 inscriptos de los que el $62 \%$ eran mujeres y un $38 \%$ hombres (Universidad Nacional de Córdoba, 2016). Del total de la muestra del presente estudio el 91\% (771) concurrían a la universidad y un 9\% (77) no completó este dato. Entre quienes indicaron que concurrian a la universidad, el $78 \%$ cursaba en la Universidad Nacional de Córdoba (UNC) y el $22 \%$ en universidades privadas de la provincia de Córdoba. Con respecto a las facultades de procedencia de los participantes, la distribución de la muestra fue la siguiente: Facultad de Artes (2.9\%), Ciencias Agropecuarias (7\%), Facultad de Arquitectura, Urbanismo y Diseño (2.7\%), Facultad de Derecho y Ciencias Sociales (10,7\%), Facultad de Ciencias Económicas (5.5\%), Facultad de Ciencias Exactas, Físicas y Naturales (12.7\%), Facultad de Filosofia y 
Humanidades (4.1\%), Facultad de Lenguas (8\%), Facultad de Matemática, Astronomía y Física (3.2\%), Facultad de Ciencias Médicas (14.2\%), Facultad de Odontología (2.2\%), Facultad de Psicología (14.5\%), Facultad de Ciencias Químicas $(2.3 \%)$, otra $(1 \%)$. Con respecto al nivel socioeconómico, se les preguntó a los participantes acerca de la percepción que ellos poseían sobre su situación económica. El resultado indicó que el $8 \%$ calificó su situación económica como muy buena, el 54\% como buena, el 32\% como regular, el 5\% como mala y el $1 \%$ como muy mala.

\section{Instrumentos}

Autocontrol. Para evaluar este constructo se utilizó la Escala de Autocontrol Breve (BSCS; Tangney et al., 2004) en su versión adaptada por Garrido, Cupani y Arbach (2017). Esta escala está compuesta por 13 ítems que poseen un formato de respuesta tipo Likert con 5 opciones que van desde 1 (para nada de acuerdo) hasta 5 (totalmente de acuerdo). La confiabilidad evaluada por los autores mediante el coeficiente alfa de Cronbach fue igual a .85. En relación con la validez, Tangney et al. (2004) reportaron que las personas con puntajes más elevados en la escala BSCS presentan un mejor ajuste psicológico, mejores habilidades interpersonales y menos problemas de comportamiento como los atracones de comida o el consumo excesivo de alcohol.

Consumo de sustancias. Los participantes respondieron seis preguntas que permitieron evaluar la frecuencia de consumo de alcohol y de drogas, utilizando una escala de respuesta de cinco puntos 1 (nunca lo hice), 2 (lo hice una vez), 3 (lo hice 2 a 3 veces), 4 (lo hice 4 a 6 veces), 5 (lo hice 6 veces o más veces). Se utilizaron seis preguntas para medir la frecuencia de consumo de alcohol (en bebidas de 1-alta y 2-mediana/baja graduación y 3-consumo hasta la ebriedad), tabaco, marihuana, cocaina/éxtasis. Para responder, los participantes utilizaron una escala de respuesta de cinco puntos, 1 (nunca lo hice), 2 (lo hice una vez), 3 (lo hice 2 a 3 veces), 4 (lo hice 4 a 6 veces), 5 (lo hice 6 veces o más veces). Para el análisis de evidencia de validez de grupos contrastados, se recodificaron estas respuestas en dos categorias: con [opciones de respuesta 2 a 5] y sin consumo. El objetivo de la recodificación fue reducir la variabilidad interindividual al interior de los grupos y al mismo tiempo marcar las posibles diferencias entre los grupos con ausencia de consumo de aquellos con presencia de consumo de sustancias. Cabe mencionar que la selección de estas preguntas se hizo teniendo en cuenta investigaciones previas que estudiaron el nivel de asociación entre el constructo autocontrol y comportamientos, como ser consumo de alcohol y consumo de sustancias (Duckworth \& Kern, 2011).

\section{Procedimiento}

La escala se aplicó a estudiantes universitarios de forma on-line, a través de la plataforma virtual Lime Survey que ofrece el servidor de encuestas de la UNC. La invitación para participar se realizó por redes sociales oficiales de las facultades de la UNC. Los estudiantes debieron dar su consentimiento como paso inicial para luego poder acceder a la visualización de la escala. Los procedimientos del estudio, incluyendo los aspectos éticos, fueron aprobados por la Comisión Evaluadora de Proyectos de la Secretaría de Ciencia y Técnica de la UNC. El tiempo requerido para completar la totalidad de las preguntas se estipuló entre 20 y 25 minutos aproximadamente. 


\section{Análisis de Datos}

Para la preparación de los datos, se utilizó el programa estadístico SPSS para Windows, versión 19.0. En primer lugar, se identificaron los casos atípicos univariados y multivariados mediante la prueba de distancia de Mahalanobis $(p<.001)$ (Tabachnick \& Fidell, 2001). Además, se calculó la media, desviación estándar, distribución de frecuencia, asimetría y curtosis. Como criterio para evaluar los índices de asimetría y curtosis se consideró como excelentes a los valores entre \pm 1.00 , y adecuados a los inferiores a \pm 2.00 (George \& Mallery, 2012).

Posteriormente se realizó un AFC para examinar si los ítems se agrupaban en las dimensiones factoriales sugeridas por los modelos propuestos por Tangney et al. (2004), Ferrari et al. (2009), De Ridder et al. (2011), Maloney et al. (2004) y Morean et al. (2014). Para ello se organizaron las matrices de datos considerando la cantidad de items que componian la BSCS, como así también el agrupamiento de los ítems que componían cada dimensión factorial según cada modelo teórico. Para el AFC se utilizó como método de estimación la técnica de robustos mínimos cuadros ponderados (WLS, por sus siglas en inglés), considerada la más oportuna para datos observados discretos (Muthén \& Kaplan, 1985). Para examinar el ajuste estadístico de los modelos rivales se utilizó el software estadístico Mplus versión 6.12 (Muthén \& Muthén, 2011). Para evaluar el ajuste de los modelos, se utilizaron los estadísticos Chi-Cuadrado, el Índice de Ajuste Comparativo $(C F I)$, el Índice de Tucker-Lewis (TLI), el Error Cuadrático Medio de Aproximación (RMSEA), y la ponderada Media Cuadrática Residual (WRMR). Los valores entre .90 y .95 para el CFI y $T L I$ son considerados como ajustes aceptables, y los superiores a .95 son considerados excelentes, mientras que se esperan valores entre .05 y .08 para el RMSEA, y menores a 1.00 para WRMR (Yu \& Muthén, 2002).

Para el estudio de consistencia interna se calculó el coeficiente Omega (McDonald, 1999), que, a diferencia del coeficiente alfa de Cronbach, emplea las cargas factoriales, que son la suma ponderada de las variables estandarizadas, lo que otorga mayor estabilidad a los cálculos (Zinbarg, Revelle, Yovel, \& Li, 2005; Zinbarg, Yovel, Revelle, \& McDonald, 2006). Valores entre .70 y .90 se consideran aceptables (Nunnally, 1978).

Por último, se realizó un estudio de evidencia de validez de grupos contrastados comparando los puntajes medios entre quienes consumian y quienes no consumian bebidas alcohólicas o drogas ilegales mediante una prueba $t$ para muestras independientes. Para estimar el tamaño del efecto se utilizó la d de Cohen (1988), donde valores de .20, .50 y .80 indican tamaño del efecto pequeño, medio y grande, respectivamente

\section{RESULTADOS}

\section{Análisis de ítems}

La muestra inicial estuvo compuesta por 895 casos, de los cuales se identificaron 34 casos atípicos mediante el cálculo de puntuaciones estándar y 13 casos atípicos multivariados. Estos casos fueron eliminados y la muestra quedó conformada por 848 participantes. Con respecto a los valores del índice de asimetría, se observó que los 13 items de la escala presentaron valores comprendidos entre +1 y -1 , considerados por la literatura como excelentes, 
(George \& Mallery, 2012). Con respecto a los valores del índice de curtosis, 10 items presentaron valores considerados excelentes (entre -1 y +1 ) y 3 items presentaron valores considerados adecuados.

\section{Análisis factorial confirmatorio}

Los resultados del AFC indicaron que el modelo propuesto por Maloney et al. (2012) presentó inicialmente mejores indices de ajuste que los modelos propuestos por Tangney et al. (2004), Ferrari et al. (2009) y De Ridder et al. (2011), cuyos índices de ajuste no fueron adecuados (ver tabla 1). Además, se evaluó si existía relación entre las dimensiones de los modelos bidimensionales propuestos y se encontró que, en el caso del modelo de Maloney et al., el nivel de asociación entre las dimensiones fue igual a -.81, mientras que en los modelos de Morean et al.(2014) Ferrari et al. y De Ridder et al., los niveles de asociación fueron de -.69, -.73 y -1 respectivamente.

Considerando estos resultados, se procedió al análisis de los índices de modificación de los ítems. Se corroboró que existian altas covariaciones entre algunos de ellos, por lo que estas correlaciones fueron incluidas en el modelo. En el caso del modelo de Tangney et al. (2004) se correlacionaron tres pares de items; en el caso del modelo propuesto por Ferrari et al. (2009), se asociaron dos pares de items; y en el de De Ridder et al. (2011), se asociaron dos items. A partir de estas modificaciones, el modelo propuesto por Tangney et al. fue el que presentó mejores índices de ajuste de los datos (ver tabla 1). Se evaluó la diferencia entre el modelo de Maloney et al. (2012) y la versión breve de Tangney et al. mediante un análisis de modelos rivales. Como resultado, se encontró que no existen diferencias significativas $\left(\Delta \chi^{2}[43, N=848]=221.39\right.$, $p>$.05).

\section{Análisis de Consistencia Interna}

El índice de confiabilidad Omega (McDonald, 1999) indicó valores considerados adecuados para la dimensión Autocontrol (Tangney et al., 2004), Autodisciplina General (Ferrari et al., 2009) e Inhibición (De Ridder et al., 2011). Por el contrario, el resto de las dimensiones propuestas en los modelos teóricos no presentaron valores considerados aceptables en el índice de confiabilidad (ver tabla 1).

\section{Evidencia de validez de grupos contrastados}

Se observó que la mayoría de las dimensiones factoriales permitieron determinar diferencias significativas en el constructo autocontrol entre los grupos de participantes que informaron haber consumido alguna de las seis sustancias evaluadas de los que no consumian. Sin embargo, la dimensión Iniciación del modelo propuesto por De Ridder et al., (2011), no permitió valorar diferencias en el consumo de bebidas con alta graduación alcohólica como la cerveza o el vino y el consumo de tabaco. Por su parte, las dimensiones Impulsividad (Maloney et al., 2012), Autodisciplina y Control de los Impulsos (Morean et al., 2014) no permitieron valorar diferencias en el consumo de bebidas alcohólicas más comunes como la cerveza o el vino. Con respecto al tamaño del efecto de las diferencias entre los grupos, se encontró que los valores variaron entre $\mathrm{d}=0,05$ (tamaño pequeño) hasta $d=.86$ (tamaño del efecto grande), ambos valores, pertenecientes a las dimensiones Iniciación e Inhibición (De Ridder et al., 2011), respectivamente (ver Tablas 2 y 3). 
Garrido, J. S., Morán, V., Azpilicueta, A. E., Cortez, F., Arbach, K. \& Cupani, M. / Psicodebate, 18(2), 26 37.

Tabla 1.

Cargas factoriales estandarizadas de los items, coeficientes de confiabilidad e índices de ajuste estadístico de los modelos uni y bidimensionales de la BSCS.

\begin{tabular}{|c|c|c|c|c|c|c|c|c|c|}
\hline \multirow[t]{2}{*}{ Ítems de la BSCS } & \multirow{2}{*}{$\begin{array}{c}\text { Modelo } \\
\text { Tangney }\end{array}$} & \multicolumn{2}{|c|}{$\begin{array}{l}\text { Modelo } \\
\text { Ferrari } \\
\end{array}$} & \multicolumn{2}{|c|}{$\begin{array}{l}\text { Modelo de } \\
\text { De Ridder }\end{array}$} & \multicolumn{2}{|c|}{$\begin{array}{l}\text { Modelo } \\
\text { Maloney }\end{array}$} & \multicolumn{2}{|c|}{$\begin{array}{l}\text { Modelo } \\
\text { Morean }\end{array}$} \\
\hline & & AG & $\mathrm{Cl}$ & INH & INI & $\mathbf{R}$ & I & A & $\mathbf{C l}$ \\
\hline Soy bueno para resistir tentaciones & .40 & - & .50 & .40 & - & .44 & - & .53 & - \\
\hline $\begin{array}{l}\text { Me cuesta cambiar hábitos } \\
\text { inadecuados }\end{array}$ & .53 & .54 & - & $.49^{*}$ & - & $.58^{*}$ & - & - & - \\
\hline Soy perezoso & .56 & .56 & - & - & $.54^{*}$ & - & - & - & - \\
\hline $\begin{array}{l}\text { Digo cosas inapropiadas } \\
\text { Si son divertidas, puedo hacer }\end{array}$ & .41 & .41 & - & - & - & - & - & - & - \\
\hline $\begin{array}{l}\text { cosas que son malas o negativas } \\
\text { para mí }\end{array}$ & .54 & .54 & - & $.54^{*}$ & - & - & .52 & - & .52 \\
\hline $\begin{array}{l}\text { Me gustaría tener más } \\
\text { autodisciplina }\end{array}$ & .50 & .51 & - & - & - & $.56^{*}$ & - & - & - \\
\hline $\begin{array}{l}\text { El placer y la diversión a veces me } \\
\text { distraen y no hago mi trabajo }\end{array}$ & .68 & .69 & - & $.71^{*}$ & - & - & .61 & - & .63 \\
\hline $\begin{array}{l}\text { Tengo problemas para } \\
\text { concentrarme }\end{array}$ & .65 & .66 & - & - & $.66^{*}$ & - & - & - & - \\
\hline $\begin{array}{l}\text { Soy capaz de trabajar } \\
\text { efectivamente hacia objetivos a } \\
\text { largo plazo }\end{array}$ & .43 & - & .54 & - & .43 & - & - & .55 & - \\
\hline $\begin{array}{l}\text { A veces no puedo dejar de hacer } \\
\text { algo, incluso si sé que es un error }\end{array}$ & .59 & .59 & - & $.58^{*}$ & - & - & .68 & - & .65 \\
\hline $\begin{array}{l}\text { A menudo actúo sin pensar en } \\
\text { todas las alternativas }\end{array}$ & .51 & .52 & - & - & $.50^{*}$ & - & .57 & - & .57 \\
\hline $\begin{array}{l}\text { Rechazo cosas que son malas o } \\
\text { negativas para mí }\end{array}$ & .40 & - & .49 & .42 & - & - & - & - & - \\
\hline $\begin{array}{l}\text { Las personas podrían decir de mí } \\
\text { que tengo una disciplina férrea }\end{array}$ & .27 & - & .34 & - & - & .27 & - & .36 & - \\
\hline$\omega$ & .81 & .80 & .53 & .70 & 62 & .53 & .69 & .47 & .69 \\
\hline$\chi^{2}$ & 512.979 & \multicolumn{2}{|c|}{465.245} & \multicolumn{2}{|c|}{353.657} & \multicolumn{2}{|c|}{100.647} & \multicolumn{2}{|c|}{86.847} \\
\hline $\mathrm{CFI}$ & .868 & \multicolumn{2}{|c|}{.882} & \multicolumn{2}{|c|}{.881} & \multicolumn{2}{|c|}{.942} & \multicolumn{2}{|c|}{.933} \\
\hline TLI & .842 & \multirow{2}{*}{\multicolumn{2}{|c|}{.856}} & \multirow{2}{*}{\multicolumn{2}{|c|}{$\begin{array}{l}.842 \\
105\end{array}$}} & \multicolumn{2}{|c|}{.915} & \multicolumn{2}{|c|}{.893} \\
\hline RMSEA & .090 & & & \multirow{2}{*}{\multicolumn{2}{|c|}{$\begin{array}{l}.105 \\
1.722\end{array}$}} & \multirow{2}{*}{\multicolumn{2}{|c|}{$\begin{array}{l}.071 \\
1.068\end{array}$}} & \multirow{2}{*}{\multicolumn{2}{|c|}{$\begin{array}{l}.082 \\
1.103\end{array}$}} \\
\hline WRMR & 1.727 & \multicolumn{2}{|c|}{$\begin{array}{l}.000 \\
1.630\end{array}$} & & & & & & \\
\hline \multicolumn{10}{|l|}{ Modelo Post-hoc } \\
\hline$x^{2}$ & 322.045 & \multicolumn{2}{|c|}{352.023} & \multicolumn{2}{|c|}{278.973} & & & & \\
\hline CFI & 0.923 & \multicolumn{2}{|c|}{0.915} & \multicolumn{2}{|c|}{0.908} & & & & \\
\hline TLI & 0.904 & \multicolumn{2}{|c|}{0.893} & \multicolumn{2}{|c|}{0.875} & & & & \\
\hline RMSEA & 0.070 & \multirow{2}{*}{\multicolumn{2}{|c|}{$\begin{array}{l}0.074 \\
1.401\end{array}$}} & \multirow{2}{*}{\multicolumn{2}{|c|}{$\begin{array}{l}0.094 \\
1.520\end{array}$}} & & & & \\
\hline WRMR & 1.348 & & & & & & & & \\
\hline
\end{tabular}

\section{DISCUSIÓN}

Debido a que en nuestro contexto se detectó una carencia de instrumentos que permitan evaluar el constructo autocontrol, y en función de que la BSCS es una de las escalas más utilizadas internacionalmente (De Ridder et al, 2012), el principal objetivo del presente estudio fue evaluar la estructura factorial, la consistencia interna y la validez de la BSCS, considerando la organización de los ítems propuestos por diferentes modelos teóricos en una muestra de estudiantes universitarios de la Ciudad de Córdoba (Argentina). 
Tabla 2.

Media (M) y desviación estándar (DE), Prueba t, nivel de significación y d de Cohen de la Escala Breve de Autocontrol según el consumo de alcohol.

\begin{tabular}{|c|c|c|c|c|c|c|c|}
\hline & \multicolumn{7}{|c|}{$\begin{array}{c}\text { ¿Consumiste bebidas alcohólicas con alta graduación (por ej., } \\
\text { tequila, whisky, vodka o ginebra) antes de los } 18 \text { años? }\end{array}$} \\
\hline & \multicolumn{2}{|c|}{ Consume } & \multicolumn{2}{|c|}{ No consume } & \multirow[b]{2}{*}{$t$} & \multirow[b]{2}{*}{$p$} & \multirow[b]{2}{*}{$d$} \\
\hline & $M$ & $D E$ & $M$ & $D E$ & & & \\
\hline $\begin{array}{l}\text { Tangney } \\
\text { BSCS } \\
\text { Maloney }\end{array}$ & 41.42 & 8.11 & 44.21 & 8.64 & -4.14 & $<.001$ & 0.34 \\
\hline $\mathrm{M}$ & 11.35 & 3.11 & 12.18 & 3.22 & -3.22 & $<.001$ & -0.26 \\
\hline \multirow[t]{4}{*}{ I } & 10.57 & 3.34 & 9.60 & 3.31 & 3.55 & $<.001$ & 0.29 \\
\hline & \multicolumn{7}{|c|}{$\begin{array}{l}\text { ¿Consumiste otras bebidas alcohólicas (por ej., cerveza o vino) } \\
\text { antes de los } 18 \text { años? }\end{array}$} \\
\hline & \multicolumn{2}{|c|}{ Consume } & \multicolumn{2}{|c|}{ No consume } & & & \\
\hline & $M$ & $D E$ & $M$ & $D E$ & $t$ & $p$ & $d$ \\
\hline $\begin{array}{l}\text { Tangney } \\
\text { BSCS } \\
\text { Maloney }\end{array}$ & 41.74 & 8.19 & 44.29 & 8.82 & -2.93 & $<.001$ & -0.31 \\
\hline M & 11.42 & 3.17 & 12.35 & 2.96 & -2.81 & .005 & -0.30 \\
\hline \multirow[t]{4}{*}{1} & 10.43 & 3.31 & 9.79 & 3.63 & 1.84 & .067 & 0.19 \\
\hline & \multicolumn{7}{|c|}{$\begin{array}{l}\text { ¿Te emborrachaste intencionadamente solo por diversión (a } \\
\text { cualquier edad)? }\end{array}$} \\
\hline & \multicolumn{2}{|c|}{ Consume } & \multicolumn{2}{|c|}{ No consume } & & & \\
\hline & $M$ & $D E$ & $M$ & $D E$ & $t$ & $p$ & $d$ \\
\hline $\begin{array}{l}\text { Tangney } \\
\text { BSCS } \\
\text { Maloney }\end{array}$ & 41.11 & 8.07 & 45.37 & 8.30 & -6.35 & $<.001$ & -0.52 \\
\hline $\mathrm{M}$ & 11.27 & 3.10 & 12.46 & 3.16 & -4.60 & $<.001$ & -0.38 \\
\hline I & 10.69 & 3.30 & 9.17 & 3.29 & 5.58 & $<.001$ & 0.46 \\
\hline
\end{tabular}

Para obtener evidencia de validez de la estructura interna de la BSCS, se evaluaron los indices de bondad de ajuste de los diferentes modelos propuestos y se encontró, al igual que en el estudio realizado por Lindner et al. (2015), que el modelo de Maloney et al. (2012) presenta los mejores índices de ajuste. En este modelo, los autores proponen que la escala se encuentra compuesta por dos facetas que permiten medir, por una parte, la iniciación de una conducta tendiente a postergar la satisfacción de las necesidades inmediatas -factor Restricción-y, por el otro, el comportamiento espontáneo guiado por la intuición y la heurística -factor Impulsividad- (Maloney et al, 2012). Considerando este resultado, se analizó el nivel de asociación que existía entre las dos dimensiones propuestas por Maloney et al. (2012) y se encontró que entre ellas existía una correlación de Pearson igual a -.81. Este grado de correlación y su direccionalidad en sentido negativo, a la luz de los criterios de interpretación propuestos en la literatura (Bentler, 2006), indican que estos factores permiten medir dos dimensiones diferenciadas entre sí, pero que se encuentran contenidas por un factor de orden superior que podría ser denominado, siguiendo con la nomenclatura utilizada por los creadores de la BSCS, como autocontrol. 
Garrido, J. S., Morán, V., Azpilicueta, A. E., Cortez, F., Arbach, K. \& Cupani, M. / Psicodebate, 18(2), 26 37.

\begin{tabular}{|c|c|c|c|c|c|c|c|}
\hline \multirow[b]{4}{*}{$\begin{array}{l}\text { Tangney } \\
\text { BSCS }\end{array}$} & \multicolumn{7}{|c|}{$\begin{array}{l}\text { ¿Consumiste regularmente productos con tabaco (por ej., cigarrillos } \\
\text { o pipa)? }\end{array}$} \\
\hline & \multicolumn{2}{|c|}{ Consume } & \multicolumn{2}{|c|}{ No consume } & \multirow[b]{2}{*}{$t$} & \multirow[b]{2}{*}{$p$} & \multirow[b]{2}{*}{$d$} \\
\hline & $M$ & $D E$ & $M$ & $D E$ & & & \\
\hline & 40.77 & 8.38 & 43.26 & 8.06 & -4.40 & $<.001$ & -0.30 \\
\hline \multirow[t]{4}{*}{$\begin{array}{l}\text { Maloney } \\
\text { M } \\
\text { I }\end{array}$} & $\begin{array}{l}11.07 \\
10.72\end{array}$ & $\begin{array}{l}3.12 \\
3.34\end{array}$ & $\begin{array}{l}11.97 \\
10.01\end{array}$ & $\begin{array}{l}3.12 \\
3.34\end{array}$ & $\begin{array}{r}-4.22 \\
3.08\end{array}$ & $\begin{array}{l}<.001 \\
<.001\end{array}$ & $\begin{array}{r}-0.29 \\
0.21\end{array}$ \\
\hline & \multicolumn{7}{|c|}{$\begin{array}{l}\text { ¿Consumiste regularmente productos con tabaco (por ej., cigarrillos } \\
\text { o pipa)? }\end{array}$} \\
\hline & \multicolumn{2}{|c|}{ Consume } & \multicolumn{2}{|c|}{ No consume } & & & \\
\hline & $M$ & $D E$ & $M$ & $D E$ & $t$ & $p$ & $d$ \\
\hline $\begin{array}{l}\text { Tangney } \\
\text { BSCS }\end{array}$ & 40.77 & 8.38 & 43.26 & 8.06 & -4.40 & $<.001$ & -0.30 \\
\hline $\begin{array}{l}\text { Maloney } \\
\text { M } \\
\text { I }\end{array}$ & $\begin{array}{l}11.07 \\
10.72\end{array}$ & $\begin{array}{l}3.12 \\
3.34\end{array}$ & $\begin{array}{l}11.97 \\
10.01\end{array}$ & $\begin{array}{l}3.12 \\
3.34\end{array}$ & $\begin{array}{l}-4.22 \\
3.08\end{array}$ & $\begin{array}{l}<.001 \\
<.001\end{array}$ & $\begin{array}{r}-0.29 \\
0.21\end{array}$ \\
\hline & \multicolumn{7}{|c|}{ ¿Consumiste drogas blandas como marihuana? } \\
\hline & \multicolumn{2}{|c|}{ Consume } & \multicolumn{2}{|c|}{ No consume } & & & \\
\hline & $M$ & $D E$ & $M$ & $D E$ & $t$ & $p$ & $d$ \\
\hline $\begin{array}{l}\text { Tangney } \\
\text { BSCS }\end{array}$ & 40.73 & 8.41 & 43.51 & 7.95 & -4.94 & $<.001$ & -0.34 \\
\hline $\begin{array}{l}\text { Maloney } \\
\text { M } \\
\text { I }\end{array}$ & $\begin{array}{l}11.16 \\
10.87\end{array}$ & $\begin{array}{l}3.22 \\
3.40\end{array}$ & $\begin{array}{c}11.95 \\
9.79\end{array}$ & $\begin{array}{l}3.03 \\
3.22\end{array}$ & $\begin{array}{l}-3.69 \\
4.75\end{array}$ & $\begin{array}{l}<.001 \\
<.001\end{array}$ & $\begin{array}{l}-0.25 \\
0.33\end{array}$ \\
\hline & \multicolumn{7}{|c|}{ ¿Consumiste drogas duras como cocaína o éxtasis? } \\
\hline & \multicolumn{2}{|c|}{ Consume } & \multicolumn{2}{|c|}{ No consume } & & & \\
\hline & $M$ & $D E$ & $M$ & $D E$ & $t$ & $p$ & $d$ \\
\hline $\begin{array}{l}\text { Tangney } \\
\text { BSCS }\end{array}$ & 36.08 & 7.98 & 42.52 & 8,16 & -6.00 & $<.001$ & -0.79 \\
\hline $\begin{array}{l}\text { Maloney } \\
\text { M } \\
\text { I }\end{array}$ & $\begin{array}{c}9.66 \\
12.58\end{array}$ & $\begin{array}{l}3.24 \\
3.02\end{array}$ & $\begin{array}{l}11.68 \\
10.18\end{array}$ & $\begin{array}{l}3,10 \\
3,32\end{array}$ & $\begin{array}{l}-4.93 \\
5.52\end{array}$ & $\begin{array}{l}<.001 \\
<.001\end{array}$ & $\begin{array}{c}-0.65 \\
0.73\end{array}$ \\
\hline
\end{tabular}

A partir del análisis de validez de grupos contrastados, se observó que los participantes del grupo que indicó haber consumido al menos una vez alguna de las sustancias evaluadas presentó un valor promedio superior en el factor Impulsividad con respecto al grupo de no consumidores, mientras que cuando se evaluó el factor Restricción se observó lo contrario. Estos resultados coinciden con estudios previos que informaron que en grupos en los que no se consume alcohol existe un mayor nivel de autocontrol, mientras que en grupos en los que sí se consume alcohol existe un mayor nivel de impulsividad (Tangney et al., 2004). Sin embargo, cuando se evaluó el tamaño del efecto de dichas diferencias de medias, lo resultados no fueron contundentes ya que el tamaño del efecto en la mayoría de los casos fue mediano (entre .20 y .80). Por este motivo, no se puede considerar que el modelo propuesto por Maloney et al. (2012) presente mejores cualidades para diferenciar, en cuanto a autocontrol o impulsividad, entre aquellos sujetos que consumen y aquellos sujetos que no lo hacen. Además, pese a los satisfactorios resultados que se obtuvieron en los análisis estadísticos a nivel general, no se obtuvieron 
resultados adecuados en el índice del coeficiente Omega de McDonald (Fornell \& Larcker, 1981; Nunnally, 1978).

Por otro lado, los resultados del modelo post hoc realizados sobre el modelo de la escala de Tangney et al. (2004) evidenciaron un aumento notable de los valores de ajuste estadístico, que llegaron a ser adecuados. Sumado a esto, los resultados del análisis de validez y de confiabilidad del modelo propuesto por Tangney et al. (2004) indican propiedades psicométricas superiores en este modelo con respecto a las observadas en los otros modelos evaluados en el presente trabajo. De hecho, los valores de confiabilidad encontrados son similares a los reportados en otros estudios (Lindner et al., 2015; Morean et al., 2014). La validez de este modelo se reafirma en diferentes muestras y culturas, lo cual sustenta la posibilidad de su utilización unidimensional (Brevers, Foucart, Verbanck, \& Turel, 2017; Unger, Bi, Xiao, \& Ybarra, 2016). Por último, cabe mencionar que el resto de los modelos analizados no presentaron ajustes estadísticos adecuados. Asimismo, los valores de confiabilidad en su mayoría tampoco fueron satisfactorios.

\section{Limitaciones}

Algunas de las limitaciones del presente estudio se relacionan con la muestra utilizada. Por un lado, está compuesta en su mayoría por mujeres, lo que limita la posibilidad de evaluar si los resultados obtenidos se encuentran determinados o no por el sexo de los participantes. Por otro lado, si bien la muestra objetivo de nuestro estudio fueron estudiantes universitarios, cabe mencionar que los resultados obtenidos carecen de fiabilidad para ser utilizados en otras muestras que puedan ser de interés, como adolescentes, adultos mayores, pacientes clínicos o incluso en jóvenes con edades comprendidas entre los 18 y 30 años, pero que no asistan a la universidad. Asimismo, los resultados obtenidos en este estudio surgieron de la administración en una sola muestra, por lo cual no se posee información acerca de la estabilidad de las estructuras factoriales propuestas por los modelos teóricos en muestras sucesivas e independientes. Por último, en el presente estudio no se evaluó la presencia de deseabilidad social en los participantes.

\section{Futuras Líneas de Investigación}

Futuros trabajos de investigación deberian comprobar si la escala con dos facetas permite valorar efectivamente la dimensión autocontrol en muestras de participantes con características diferentes, como, por ejemplo, en muestras de adolescentes en conflicto con la ley penal, en pacientes jóvenes con patologías psiquiátricas, como el consumo abusivo de sustancias, o en muestras de adultos. Asimismo, se debería ahondar en el estudio del poder predictivo a largo plazo de la escala, mediante un diseño longitudinal. Además, sería importante incluir medidas que permitan obtener datos de validez discriminante de las facetas obtenidas y realizar estudios de validación cruzada para determinar la estabilidad de la estructura factorial de la BSCS en diferentes muestras, de forma sucesiva e independiente.

En sintesis, a partir de los resultados obtenidos se puede inferir que el modelo post hoc unidimensional propuesto por Tangney, et al. (2004) es la opción que mejores propiedades psicométricas presentó, por lo cual es un instrumento adecuado para ser utilizado con fines de investigación en muestras de estudiantes universitarios argentinos. 


\section{REFERENCIAS}

Bentler, P. M. (2006). EQS 6 Structural equations program manual. Encino, CA: Multivariate Software, Inc.

Brevers, D., Foucart, J., Verbanck, P., \& Turel, O. (2017). Examination of the validity and reliability of the French versionof the Brief Self-Control Scale. Canadian Journal of Behavioural Science, 49(4), 243-250. https: / / doi.org/10.1037/cbs0000086

Cohen, J. (1988). Statistical power analysis for the behavioral sciences. Second Edition. Hillsdate, NJ: LEA. https://doi.org/10.1002/bs.3830330104

Cupani, M. (2012). Análisis de ecuaciones estructurales: conceptos, etapas de desarrollo y un ejemplo de aplicación. Revista Tesis, 1, 186-199.

De Ridder, D., De Boer, B., Lugtig, P., Bakker, A., \& Van Hooft, E. (2011). Not doing bad things is not equivalent to doing the right thing: Distinguishing between inhibitory and initiatory self-control. Personality and Individual Differences, 50, 1006-1011. https://doi.org/10.1016/j.paid.2011.01.015

De Ridder, D., Lensvelt-Mulders, G., Finkenauer, C. F., Stok, M., \& Baumeister, R. F. (2012).Taking stock of self-control: A meta-analysis of how trait self-control relates to a wide range of behaviors. Personality and Social Psychology Review, 16, 76-99. https://doi.org/10.1177/1088868311418749

Duckworth, A. L., Gendler, T. S., \& Gross, J. J. (2016). Situational strategies for self-control. Perspectives on Psychological Science, 11(1), 35-55. https://doi.org/10.1177/1745691615623247

Duckworth, A. L. \& Kern, M. L. (2011). A meta-Analysis of the convergent validity of self-control measures. Journal of Research in Personality, 45, 259-268. https://doi.org/10.1016/j.jrp.2011.02.004

Ferrari, J. R., Stevens, E. B., \& Jason, L. A. (2009). The relationship of self-control and abstinence maintenance: An exploratory analysis of self-regulation. Journal of Groups in Addiction and Recovery, 4, 32-41. https://doi.org/10.1080/15560350802712371

Fornell, C. \& Larcker, D. F. (1981). Evaluating structural equation models with unobservable variables and measurement error. Journal of marketing research, 18(1), 39-50. https://doi.org/10.2307/3151312

Garrido, S., Cupani, M., \& Arbach, K. (2017). Aplicación del Modelo de Escala de Clasificación Para Examinar las Propiedades Psicométricas de la Self-Control Scale. Psykhe, 26(2), 1-12. https://doi.org/10.7764/psykhe.26.2.980

George, D. \& Mallery, P. (2012). IBM SPSS Statistics 21 step by step: A simple guide and reference (12th ed.). Boston, MA: Pearson.

Job, V., Friese, M., \& Bernecker, K. (2015). Effects of practicing self-control on academic performance. Motivation Science, 1(4), 219-232. https://doi.org/10.1037/mot0000024

Konttinen, H., Haukkala, A., Sarlio-Lähteenkorva, S., Silventoinen, K., \& Jousilahti, P. (2009). Eating styles, self-control and obesity indicators. The moderating role of obesity status and dieting history on restrained eating. Appetite, 53(1), 131-134. https://doi.org/10.1016/j.appet.2009.05.001

Lindner, C., Nagy, G., \& Retelsdorf, J. (2015). The dimensionality of the Brief Self-Control Scale-An evaluation of unidimensional and multidimensional applications. Personality and Individual Differences, 86, 465-473. https://doi.org/10.1016/j.paid.2015.07.006

Ludwig, V. U., Stelzel, C., Krutiak, H., Prunkl, C. E., Steimke, R., Paschke, L. M., ... \& Walter, H. (2013). Impulsivity, self-control, and hypnotic suggestibility. Consciousness and cognition, 22(2), 637-653. https://doi.org/10.1016/j.concog.2013.04.001

Maloney, P. W., Grawitch, M. J., \& Barber, L. K. (2012). The multi-factor structure of the Brief Self-Control Scale: Discriminant validity of restraint and impulsivity. Journal of Research in Personality, 46(1), 111115. https://doi.org/10.1016/j.jrp.2011.10.001

Malouf, E., Stuewig, J., \& Tangney, J. (2012). Self-control and jail inmates' substance misuse post-release: Mediation by friends' substance use and moderation by age. Addictive Behaviors, 37(11), 1198-1204. https://doi.org/10.1016/j.addbeh.2012.05.013

McDonald, R. P. (1999). Test theory: A unified treatment. Mahwah, NJ: Erlbaum.

Morean, M. E., DeMartini, K. S., Leeman, R. F., Pearlson, G. D., Anticevic, A., Krishnan-Sarin, S., ... \& O'Malley, S. S. (2014). Psychometrically improved, abbreviated versions of three classic measures of impulsivity and self-control. Psychological assessment, 26(3), 1003-1020. https: / / doi.org/10.1037/pas0000003

Muthén, B., \& Kaplan, D. (1985). A comparison of some methodologies for the factor analysis of non-normal Likert variables. British Journal of Mathematical and Statistical Psychology, 38, 171-189. https://doi.org/10.1111/j.2044-8317.1985.tb00832.x

Muthén, L. K., \& Muthén, B. O. (2011). Mplus user's guide (5th ed.) Los Angeles, CA: Muthén \& Muthén.

Nunnally, J. C. (1978). Psychometric theory (2nd Ed.). New York: McGraw-Hill.

Prado, C. E., Treeby, M. S., \& Crowe, S. F. (2015). Examining relationships between facial emotion recognition, self-control, and psychopathic traits in a non-clinical sample. Personality and Individual Differences, 80, 22-27. https://doi.org/10.1016/j.paid.2015.02.013

Tabachnick, B. G., Fidell, L. S., \& Osterlind, S. J. (2001). Using multivariate statistics. Boston: Allyn and Bacon

Tangney, J. P., Baumeister, R. F., \& Boone, A. L. (2004). High self-control predicts good adjustment, less pathology, better grades, and interpersonal success. Journal of Personality, 72, 271-322. https: / / doi.org/10.1111/j.0022-3506.2004.00263.x

Turanovic, J. J., Reisig, M. D., \& Pratt, T. C. (2015). Risky lifestyles, low self-control, and violent victimization across gendered pathways to crime. Journal of Quantitative Criminology, 31(2), 183-206. https: / / doi.org/10.1007/s10940-014-9230-9

Unger, A., Bi, C., Xiao, Y. Y., \& Ybarra, O. (2016). The revising of the Tangney Self-Control Scale for Chinese students. PsyCh journal, 5(2), 101-116. https://doi.org/10.1002/pchj.128

Universidad Nacional de Cordoba (2016). Anuario estadístico 2016. Cordoba: Universidad Nacional de 
Córdoba.

Visser, L., de Winter, A. F., Veenstra, R., Verhulst, F. C., \& Reijneveld, S. A. (2013). Alcohol use and abuse in young adulthood: Do self-control and parents' perceptions of friends during adolescence modify peer influence? The TRAILS study. Addictive Behaviors, 38(12), 2841-2846. https://doi.org/10.1016/j.addbeh.2013.08.013

Will Crescioni, A., Ehrlinger, J., Alquist, J. L., Conlon, K. E., Baumeister, R. F., Schatschneider, C., \& Dutton, G. R. (2011). High trait self-control predicts positive health behaviors and success in weight loss. Journal of health psychology, 16(5), 750-759. https://doi.org/10.1177/1359105310390247

Yu C. Y. \& Muthén B. (2002). Evaluation of model fit indices for latent variable models with categorical and continuous outcomes (Technical Report). Los Angeles: University of California at Los Angeles, Graduate School of Education \& Information Studie

Zinbarg, R. E., Revelle, W., Yovel, I., \& Li. W. (2005). Cronbach's Alpha, Revelle's Beta, McDonald's Omega: Their relations with each and two alternative conceptualizations of reliability. Psychometrika. 70, 123133. https://doi.org/10.1007/s11336-003-0974-7

Zinbarg, R. E., Yovel, I., Revelle, W., \& McDonald, R. (2006). Estimating generalizability to a universe of indicators that all have one attribute in common: A comparison of estimators for omega. Applied Psychological Measurement, 30, 121-144. https://doi.org/10.1177/0146621605278814

Recibido 13-12-2017 | Aceptado 28-05-2018 